\title{
Safety and effectiveness of escitalopram in an 8-week open study in Chinese patients with depression and anxiety
}

This article was published in the following Dove Press journal:

Neuropsychiatric Disease and Treatment

\author{
Gang Wang,' Xiumin You, ${ }^{2}$ \\ Xueyi Wang, ${ }^{3}$ Xiufeng $\mathrm{Xu},{ }^{4}$ \\ Ludong Bai, ${ }^{5}$ Jian Xie, ${ }^{6}$ \\ Zhijian Yao, ${ }^{7}$ QiZhong Yi, ${ }^{8}$ \\ Jun $\mathrm{Ma},{ }^{9}$ Jinan Wang, ${ }^{10}$ \\ Jianmin Zhuo," Cuili Hu" \\ 'Psychiatry Department, Beijing Anding \\ Hospital, Capital Medical University, \\ National Clinical Research Center \\ for Mental Disorders, Beijing, China; \\ ${ }^{2}$ Psychiatry Department, Xianyue \\ Hospital, Xiamen, China; ${ }^{3}$ Psychiatry \\ Department, Institute of Mental \\ Health of Hebei Medical University, \\ Shijiazhuang, China; ${ }^{4}$ Psychiatry \\ Department, Kunming Medical \\ University Affiliated Hospital, Kunming, \\ China; ${ }^{5}$ Psychiatry Department, \\ Shangdong Province Mental \\ Hospital, Jinan, China; ${ }^{6}$ Psychiatry \\ Department, Hangzhou First \\ People Hospital, Hangzhou, China; \\ ${ }^{7}$ Psychiatry Department, Nanjing Brain \\ Hospital, Nanjing, China; ${ }^{8}$ Psychiatry \\ Department, Xinjiang University \\ Affiliated First Hospital, Urumqi, \\ China; ' $P$ sychiatry Department, Inner \\ Mongolia Mental Hospital, Hulun \\ Buir, China; ${ }^{10}$ Medical Department, \\ Lundbeck Pharmaceutical Information \\ Consulting Co., Ltd., Beijing, China; \\ "Medical Department, Xi'an Janssen \\ Pharmaceutical Ltd., Beijing, China
}

Correspondence: Cuili Hu Medical Department, China R\&D and Scientific Affairs, Xi'an Janssen Pharmaceutical Ltd., I9F, Tower 3, China Central Place, No. 77 JianGuo Road, Chao Yang District, Beijing 100025, China Tel +861058218709

Fax +86 I0 582I 8703 Email chu9@its.jnj.com
Background: Anxiety symptoms usually worsen depression and functional impairment. The present study was aimed to evaluate the impact of escitalopram on social function and quality of life in major depressive disorder (MDD) patients with anxiety symptoms.

Patients and methods: Adult MDD patients with functional impairment (Sheehan Disability Scale [SDS] score $\geq 9$ ) and anxiety symptoms (Hamilton Anxiety Rating Scale [HAM-A] score $\geq 14$ ) received escitalopram $(10-20 \mathrm{mg}$ /day) for 8 weeks. Symptom status was assessed by SDS, Quality of Life Enjoyment and Satisfaction Questionnaire-Short Form, Montgomery-Åsberg Depression Rating Scale (MADRS), HAM-A, and Quick Inventory of Depressive SymptomatologySelf Report scales. Safety was evaluated by treatment-emergent adverse events (TEAEs).

Results: Overall, 208 (79.7\%) of 261 enrolled patients completed the 8-week treatment. Mean (SD) SDS and Quality of Life Enjoyment and Satisfaction Questionnaire-Short Form total scores were 17.4 (5.87) and 39.3 (14.43) at baseline, which improved to $7.6(6.71)$ and $61.6(15.80)$, respectively, at week 8 . Totally, $59.2 \%$ of patients achieved functional remission (SDS $\leq 6)$ and $61.7 \%$ of patients achieved depression remission (MADRS $\leq 10)$ at week $8 ; 48.1 \%$ of patients achieved both functional and depression remission ( $\mathrm{SDS} \leq 6$ and MADRS $\leq 10)$. The change in SDS total score was positively correlated with the change in MADRS and HAM-A total scores at each visit. Patient's baseline SDS score was related with depression score (regression coefficient $=0.40582$, $p=0.0005$ ); remission of SDS was statistically related to a reduction of week 2 and week 6 HAM-A score $(p<0.0001)$ and reduction of MADRS score $(p<0.0001)$. Overall, $25.7 \%$ of patients reported $\geq 1$ TEAEs. Most frequently reported TEAEs were nausea (5.8\%), diarrhea (2.3\%), and dizziness (2.7\%). Most TEAEs were mild to moderate in severity. Four patients reported serious TEAEs, two patients reported suicide attempts, and one patient completed suicide.

Conclusion: Escitalopram (10-20 mg/day) treatment was efficacious in reducing depression, improving social function, and quality of life in MDD patients with anxiety symptoms. No new safety signals were identified.

Keywords: anxiety, Chinese, escitalopram, major depressive disorder, social function

\section{Introduction}

Major depressive disorder (MDD) is currently the second leading cause of disability in the world, causing a significant burden on individuals and society. ${ }^{1,2}$ The MDD is associated with a 23 -fold increase in the risk of social disability compared with the general population. ${ }^{3}$ The current prevalence of MDD in urban China is estimated at $6.0 \%,{ }^{4}$ which is higher than in rural areas $(2.0 \%) .{ }^{5}$ The severity of MDD is associated with increased treatment costs, unemployment, short- and long-term disabilities, work absenteeism, and attendance. ${ }^{6-8}$ 
Comorbid anxiety symptoms are common in patients with depressive disorders. ${ }^{9}$ Anxiety symptoms increase disease severity ${ }^{9}$ and often cause greater impairment of overall functioning. ${ }^{10}$ In the China, an estimated $68.9 \%$ of patients with MDD have comorbid anxiety disorders, ${ }^{11,12}$ which usually hinder treatment response and functional improvement. In the Netherlands, in a study of depression and anxiety, comorbid depression and anxiety were positively associated with the intensity of disabling or severely limiting pain. ${ }^{13}$ Similarly, in a recent open-label study from the China, patients with higher baseline depression and anxiety level took longer time to remit. ${ }^{14}$ The presence of comorbid anxiety symptoms in patients with MDD was associated with lower rates of remission ${ }^{15}$ and has been shown to interfere with treatment response. ${ }^{16}$ Data from a large multicenter antidepressant study, Sequenced Treatment Alternatives to Relieve Depression, demonstrated that high levels of anxiety predicted poorer response to antidepressant medications. It has also been established that comorbid anxiety disorders increase the risk of suicide among depressed patients. ${ }^{17}$

An association between depression severity and functional impairment is well documented in patients with MDD. ${ }^{18-20}$ The proportion of patients with severe functional impairment in MDD is higher than that reported in common physical diseases such as arthritis, chronic spinal pain, and high blood pressure. ${ }^{21}$ Functional impairment causes stressors and intensifies depression severity, which can persist even in patients with symptom remission..$^{22,23}$ Notably, returning to the predepression level of functioning is as important as symptom remission. ${ }^{24}$ As a part of recovery, restoration of the previous functioning is an essential goal of antidepressant therapy, as residual depressive symptoms are associated with a poor prognosis for relapse or recurrence and can lead to long-term psychosocial impairment. ${ }^{25-27}$ Hence, thorough remission of the function is important to achieve better prognosis, improved quality of life (QoL), and reduced health care costs in patients with MDD..$^{28,29}$

Selective serotonin reuptake inhibitors (SSRIs) are the first-line treatment for both depression and anxiety disorders. ${ }^{30}$ Escitalopram, the S-enantiomer of citalopram and the most selective of the SSRIs, has demonstrated consistent efficacy in the treatment of patients with MDD and anxiety symptoms. ${ }^{31-33}$ In a recent network meta-analysis, escitalopram was found to be the most efficacious and well-tolerated SSRI among the new generation antidepressants. ${ }^{34}$ In MDD patients with or without comorbid anxiety, escitalopram treatment was associated with improvements in symptoms of depression. ${ }^{35}$ Escitalopram also demonstrated improved psychosocial function, ${ }^{36}$ increased functioning based on the Sheehan Disability Scale (SDS) total score and lower duration of mean sick leave days compared with duloxetine in patients with MDD. ${ }^{37}$

In the present study, we explored the impact of escitalopram $10-20 \mathrm{mg} /$ day on social function and QoL in the treatment of Chinese MDD patients with anxiety symptoms over 8 weeks. The relationship between functional impairment and symptom severity, the impact factors of baseline SDS total score and end point SDS remission will be further explored.

\section{Patients and methods}

This 8-week, open-label, multicenter, single-arm, prospective study was conducted at 10 centers across the China from April 2014 to May 2015.

The study protocol was approved by an Independent Ethics Committee or an Institutional Review Board at each study center. The names of the approving ethics committees included Beijing Anding Hospital, Hebei Medical University First Affiliated Hospital, Kunming Medical University First Affiliated Hospital, Shangdong Province Mental Hospital, Hangzhou First People Hospital, Nanjing Brain Hospital, Xinjiang University First Affiliated Hospital, and Jinan University First Affiliated Hospital. The Xianyue Hospital and the Inner Mongolia Mental Hospital obtained approval from the central ethics committee. The study was conducted in accordance with the ethical principles outlined in the Declaration of Helsinki and was consistent with Good Clinical Practices and applicable regulatory requirements. Written informed consent was obtained from all patients prior to their enrolment into the study. This study is registered at ClinicalTrials.gov (NCT01870843).

\section{Patients}

\section{Inclusion criteria}

Adult patients between 18 and 65 years diagnosed with MDD according to Diagnostic and Statistical Manual of Mental Disorders, 4th edition (DSM-IV) diagnostic criteria ${ }^{38}$ were enrolled. Patients were included if they had a functional impairment (SDS score of $\geq 9$ ) and anxiety symptoms (Hamilton Anxiety Rating Scale [HAM-A] score of $\geq 14$ ) at screening and baseline.

\section{Exclusion criteria}

Patients with significant risk of suicide on clinical assessment ( $>5$ on item 10 of Montgomery-Åsberg Depression Rating Scale [MADRS]), or categorized as positive suicide idea or behavior on Columbia-Suicide Severity Rating Scale 
(C-SSRS), or had made a serious suicide attempt within the past 6 months were excluded. Patients with unstable serious illness and/or clinically significant renal or hepatic impairment, seizure disorders, patients with a history of primary or comorbid diagnoses of schizophrenia, schizoaffective, bipolar disorder, or dementia were excluded. Patients were also excluded if they were on psychoactive substances, antidepressants, anxiolytics, monoamine oxidase inhibitors, psychoactive herbal remedies, lithium, carbamazepine, and electroconvulsive therapy 2 weeks before baseline visit. Patients who had accepted adequate antidepressant treatment for 8 weeks in the past 2 months were excluded. Also, pregnant/lactating women or women planning for pregnancy were excluded.

\section{Treatment}

Patients received escitalopram oxalate $10 \mathrm{mg}$ /day for 1 week, which was adjusted to a maximum dose of $20 \mathrm{mg}$ /day depending on the patient's treatment response and tolerance.

\section{Allowed medications}

Benzodiazepines for the treatment of insomnia at baseline could be continued up to the first week, while non-benzodiazepine hypnotics were allowed for insomnia treatment during the entire treatment period. Other psychotropic drugs were not allowed during the study.

\section{Outcome measures}

Patients' social function was evaluated by $\operatorname{SDS}^{39}$ and QoL was evaluated by Quality of Life Enjoyment and Satisfaction Questionnaire-Short Form [Q-LES-Q-SF] ${ }^{40}$ at weeks 2, 4,6 , and 8 . The SDS used to assess disability or functional impairment is a composite of three self-rated items and uses a discretized analog (Discan) scaling metric to generate impairment scores (0-10) across three domains (work/school, social life, and family life or home responsibilities). ${ }^{39}$ The SDS total score was calculated as the sum of the scores of these three domains and can range from 0 to 30 . A severely impaired function is defined as $\mathrm{SDS} \geq 18$. Effects of treatment on improvement in functional impairment and anxiety symptoms were assessed using various definitions of remission. The self-assessment Q-LES-Q-SF scale consists of 16 items and provides subjective satisfaction and enjoyment in a series of discrete domains and life activities. Each Q-LES-Q-SF item is scored on a 5 -point scale ( $1=$ very dissatisfied, $2=$ dissatisfied, $3=$ neutral, 4=satisfied, and 5=very satisfied).

Functional remission was defined as SDS total score $\leq 6$, and work/family/social remission was defined as SDS subscale score of $\leq 2 .{ }^{41}$ Community norm samples have a mean Q-LES-Q-SF score of 78.3 (SD: 11.3), and scores within $10 \%$ of this value (like Q-LES-Q-SF $\geq 70.47$ ) were considered "within normal" QoL, which corresponds with the 75th percentile. The Q-LES-Q-SF scores $>2$ SD below community norm scores (like Q-LES-Q-SF scores $\leq 55.7$ ) were considered as "severely impaired" QoL. ${ }^{42}$

The MADRS, ${ }^{43}$ HAM-A, ${ }^{44}$ and Quick Inventory of Depressive Symptomatology-Self Report (QIDS-SR) ${ }^{45}$ were also assessed at weeks 1, 2, 4, 6, and 8. Remission of depressive symptoms was defined as MADRS total score $\leq 10$. The cut-off point for total remission on Quick Inventory of Depressive Symptomatology-Self Report (QIDS-SR) total score was $\leq 5$. Remission of anxiety symptoms was defined as HAM-A total score $\leq 7$. Depression symptom response rate, defined as $\geq 50 \%$ improvement from baseline in MADRS total score was also included as an efficacy outcome.

All raters were highly experienced, well-trained psychiatrists familiar with scales of measurements, and had received specific training about measurement tools prior to the study initiation.

\section{Exploratory analysis}

An exploratory analysis was performed to evaluate the association between change from baseline to week 8 in SDS total score and MADRS and HAM-A total scores at each visit.

Cohen's criteria were used to evaluate the substantive significance of correlations (such as large correlations: $>0.50$, medium correlations: $0.30-0.49$, and small correlations: 0.10-0.29). ${ }^{46}$ Multivariate regression analyses were performed to evaluate the impact factors of baseline SDS total score and SDS remission at end point.

\section{Statistical analyses}

Based on previous studies, it was expected that after treatment with escitalopram oxalate, Q-LES-Q-SF score would increase by 15.0 (16.0) (mean [SD]) and the SDS score would decrease by 9.77 (7.19). A total of 200 patients were considered sufficient to provide a width of Q-LES-Q-SF score increase as 4.5 and SDS score decrease as 2.0 with a two-sided 95\% CI. Assuming a $20 \%$ dropout rate, a total of 260 patients were planned to be enrolled in this study.

\section{Efficacy assessments}

Efficacy outcomes based on the full analysis set (FAS) included patients who were screened, received at least one dose of the study drug, and completed at least one assessment visit during the treatment period. For patients who did not complete 8 weeks of treatment, missing data were 
imputed using last observation carried forward. Per protocol set included patients who were screened, completed all five assessment visits during the treatment period, had good compliance, and did not had any major protocol deviation. Efficacy end points were summarized using descriptive statistics. The $95 \% \mathrm{CI}$ of the mean change from baseline in Q-LES-Q-SF and SDS scores, remission rates based on SDS, remission and response rates based on MADRS, HAM-A, and QIDS-SR scores were estimated. Depending on whether the changes from baseline scores at all treatment visits follow a normal distribution, paired $t$-test or Wilcoxon signed-rank test was used. An exploratory analysis was performed using Spearman's correlation coefficient. Multivariate regression of SDS at baseline with QoL, depression, and anxiety scores was performed using linear regression. Impact factors of SDS remission at week 8 were evaluated by single-factor logistic analysis using a stepwise selection process and then validated by multiple-factors logistic analysis. Remission at end point was considered as a dependent factor; baseline depression, anxiety, QoL, "living with family" status, reduction of depression, and anxiety at each visit were considered as covariates. The statistical analysis was performed using Statistical Analysis System version 9.1 for Windows software (SAS Institute Inc., Cary, NC, USA).

\section{Safety assessments}

Safety analyses were based on the safety set (SS), comprising all patients who took at least one dose of the study medication. Safety was assessed by evaluating the incidence and type of treatment-emergent adverse events (TEAEs), serious TEAEs, physical examination findings and vital signs (heart rate and blood pressure), C-SSRS, suicidal ideation, and suicidal behavior scores ${ }^{47}$ at all visits. All safety assessments were analyzed descriptively, and abnormal electrocardiogram (ECG) results were listed. Clinical laboratory tests and body weight were assessed at baseline and at week 8. For C-SSRS, the report was considered positive if the patient reported any of the following: active suicidal ideation with some intent to act without specific plan, active suicidal ideation with specific plan and intent, actual attempt, interrupted/aborted suicide attempt, or a behavior preparatory for making an attempt. All TEAEs were coded to preferred terms and system organ class using Medical Dictionary for Regulatory Activities, Version 17.0. Positive suicidal ideation or behavior was analyzed descriptively. Treatment compliance was based on SS and calculated as actual dose days divided by planned dose days. Good compliance was defined as treatment compliance between $80 \%$ and $120 \%$.

\section{Results \\ Patient disposition}

Of the 261 enrolled patients, 208 (79.7\%) patients completed the study. The primary reasons for discontinuations were lost to follow-up (9.2\%) and withdrawal of consent (6.9\%; Figure 1).

\section{Patient baseline demographics and clinical characteristics}

Majority of patients were women (64.7\%) in the FAS, and the mean (SD) age was 39.0 (12.75) years (Table 1). At baseline, the mean (SD) SDS total scores were 17.4 (5.9), Q-LES-Q-SF were 39.3 (14.43), MADRS were 29.1 (6.64), HAM-A were 25.6 (7.01), and QIDS-SR were 14.9 (4.29). At baseline, patients' SDS score was related to depression score (regression coefficient $=0.40582, p=0.0005$ ), and 106/241 $(43.9 \%)$ patients had a severe functional impairment while $63 / 241(26.1 \%)$ patients had both severe depression and functional impairment. About 234/241 (97.9\%) patients were classified with impaired QoL and 213/241 (89.1\%) patients with severely impaired QoL (Table 1). The mean (SD) duration of escitalopram exposure was 53.5 (14.21) days and escitalopram dose was 15.4 (3.75) mg/day. Approximately $83.0 \%$ of the patients were compliant to treatment.

\section{Prior and concomitant medication}

In the SS, all patients had depression and 9/257 (3.5\%) had hypertension. The most frequent $(>2 \%)$ prior antidepressant therapies included paroxetine $12 / 257$ (4.7\%), venlafaxine $11 / 257$ (4.3\%), sertraline $8 / 257$ (3.1\%), and deanxit $8 / 257$ (3.1\%). Clonazepam 34/257 (13.2\%) was the most common concomitant medication taken by $>2 \%$ of the patients, followed by lorazepam 14/257 (5.4\%), zopiclone 9/257 (3.5\%), Chinese herbal medicine $9 / 257$ (3.5\%), and alprazolam $7 / 257(2.7 \%)$.

\section{Efficacy SDS scores}

A positive and significant change in functioning was recorded when measured by SDS scale. In FAS, the mean (SD) SDS total scores were 17.4 (5.87) at baseline and decreased to 7.6 (6.71) at week 8 of treatment with escitalopram; change from baseline in SDS total score was statistically significant $(p<0.0001)$ at each visit. Significant improvements in work, social, and family life subscales were also reported (Table 2). The functional remission rate was $59.2 \%$ after 8 weeks of escitalopram treatment. About $48.1 \%$ of patients achieved functional and depression remission $(\mathrm{SDS} \leq 6$ 


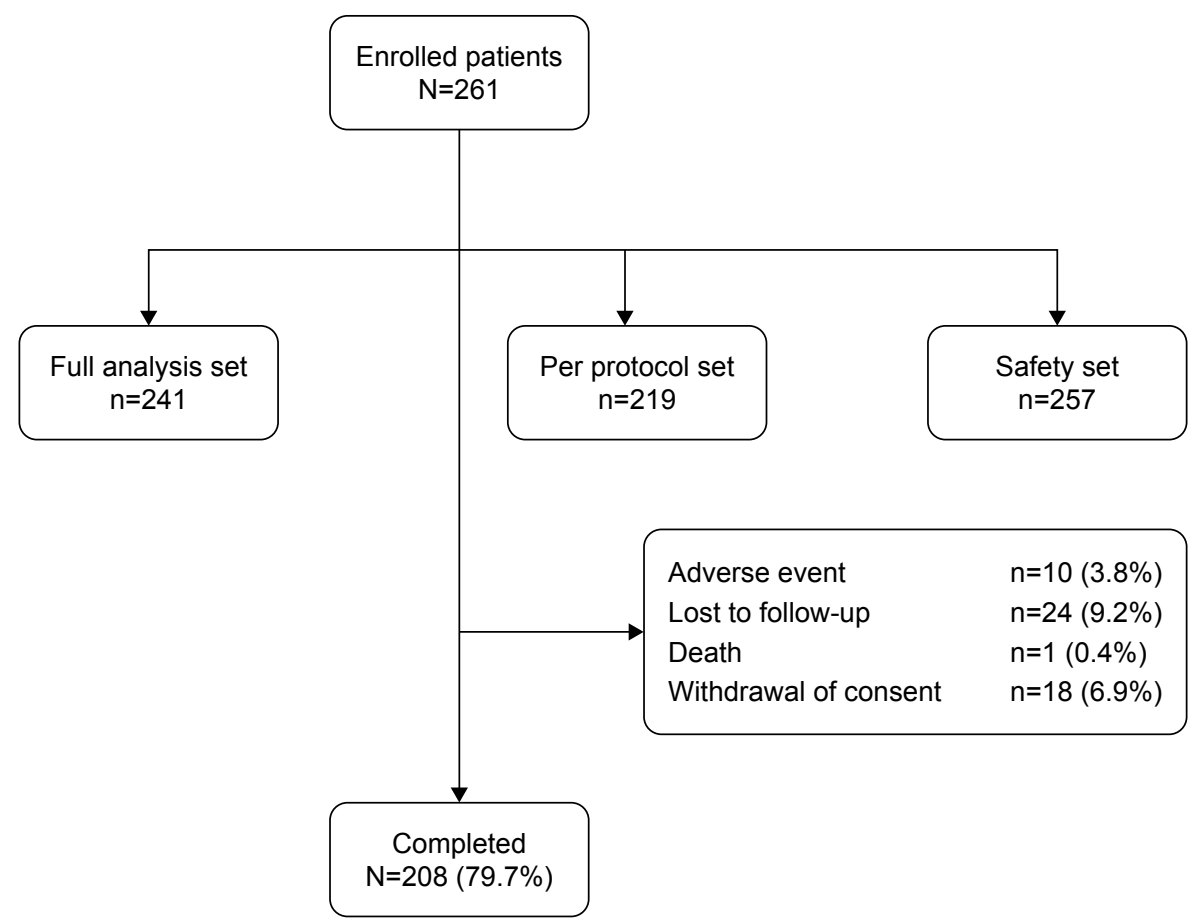

Figure I Patient disposition.

Notes: $\mathrm{N}$, total sample size; $\mathrm{n}$, total number of patients in a subset.

and MADRS $\leq 10)$. The results in per protocol set $(n=219$ patients) were consistent with that in FAS. For SDS work subscales, the percentage of remission (subscale score $\leq 2$ ) rate changed from $8.3 \%$ at baseline to $62.1 \%$ at the end of the study. For social life, the remission rate changed from $6.2 \%$ to $62.1 \%$, and for family, the remission rate changed from $8.7 \%$ to $64.7 \%$.

Multivariate linear regression of SDS at baseline with QoL, depression, and anxiety score

The baseline SDS score was related to QIDS-SR score (regression coefficient $=0.40582, p=0.0005$ ), indicating that as the baseline QIDS-SR total score increased by 1 score, the SDS increased by 0.4 scores, simultaneously. Baseline SDS score was not related to baseline QoL and anxiety scale score $(p>0.05$; Table 3$)$.

\section{Multivariate logistic regression analyses of functional remission at end point}

At the end of the study, functional remission was associated with HAM-A reduction at week 2 and week $6(\mathrm{OR}=0.984$, $p=0.0446 ; \mathrm{OR}=0.967, p=0.0001$, separately), and reduction of MADRS at week $8(\mathrm{OR}=0.951, p<0.001)$. If the change of MADRS increased by 1 score, the remission rate of SDS reduced by $3.3 \%$. When the change is negative, a higher score represents clinical worsening. Functional remission rate was not associated with baseline depression, anxiety, QoL, and social factors such as "live with family" status (Table 4).

\section{Q-LES-Q-SF scores}

The mean Q-LES-Q-SF scores improved significantly $(p<0.0001)$ as early as the second week of treatment with escitalopram (10-20 mg/day) and continued at all visits in FAS (Table 2). The mean Q-LES-Q-SF total score was 39.3 (14.43) at baseline and increased to $61.6(15.80)$ at week 8 $(p<0.0001)$. At week $8,29.7 \%$ of patients $(n=69)$ achieved normal QoL and still 70.3\% of patients $(n=163)$ had impaired QoL with $36.6 \%(n=85)$ severely impaired.

\section{MADRS scores}

The mean (SD) MADRS total score decreased significantly $(p<0.0001)$ from 29.1 (6.64) at baseline to $10.0(8.43)$ at week 8 of treatment with escitalopram in FAS (Table 2). The MADRS response and remission rate increased over time (Figure 2). Clinical response by definition (reduction of MADRS total score $\geq 50 \%$ ) was observed in $7.2 \%$ in the first week of treatment and by week 8, the response was achieved in $74.2 \%$ of patients. At week $8,61.7 \%$ of patients had achieved remission according to MADRS total score $\leq 10$. Totally, $48.1 \%$ of patients achieved depression and functional remission (MADRS total remission and SDS total remission). The Spearman's rank correlation for change 
Table I Patient baseline demographics and clinical characteristics (full analysis set)

\begin{tabular}{|c|c|}
\hline Variables & $(N=24 I)$ \\
\hline Age (years), mean (SD) ${ }^{a}$ & $39.0(12.8)$ \\
\hline Weight $(\mathrm{kg})$, mean $(\mathrm{SD})^{\mathrm{b}}$ & $62.2(10.9)$ \\
\hline Gender (women), n (\%) & $156.0(64.7)$ \\
\hline \multicolumn{2}{|l|}{ Race, n (\%) } \\
\hline Han & $233.0(96.7)$ \\
\hline Unknown & $1.0(0.4)$ \\
\hline Others & $7.0(2.9)$ \\
\hline \multicolumn{2}{|l|}{ Marital status, n (\%) } \\
\hline Married & $172.0(7 \mid .4)$ \\
\hline Single & $53.0(22.0)$ \\
\hline Divorced & $10.0(4.1)$ \\
\hline Widowed & $6.0(2.5)$ \\
\hline Alprazolam & $7(2.7)$ \\
\hline \multicolumn{2}{|l|}{ Is there excessive drinking history in the past } \\
\hline \multicolumn{2}{|l|}{12 months?, n (\%) } \\
\hline No & $241.0(100.0)$ \\
\hline \multicolumn{2}{|l|}{ Is there drug abuse history in the last } \\
\hline \multicolumn{2}{|l|}{12 months?, n (\%) } \\
\hline No & $241.0(100.0)$ \\
\hline SDS total score at baseline, mean (SD) & $17.4(5.9)$ \\
\hline Q-LES-Q-SF total score at baseline, mean (SD) & $39.3(14.43)$ \\
\hline MADRS total score, mean (SD) & $29.1(6.64)$ \\
\hline HAM-A total score, mean (SD) & $25.6(7.01)$ \\
\hline QIDS-SR total score, mean (SD) & 14.9 (4.29) \\
\hline \multicolumn{2}{|l|}{ QoL, n (\%) } \\
\hline Impaired & $234(97.9)$ \\
\hline Severely impaired & $213(89.1)$ \\
\hline
\end{tabular}

Notes: ${ }^{\mathrm{N}} \mathrm{N}=240$; ${ }^{\mathrm{N}} \mathrm{N}=239$.

Abbreviations: HAM-A, Hamilton Anxiety Scale; MADRS, Montgomery-Åsberg Depression Rating Scale; N, total sample size; $n$, total number of patients in a subset; QIDS-SR, Quick Inventory of Depressive Symptomatology-Self Report; Q-LES-QSF, Quality of Life Enjoyment and Satisfaction Questionnaire-Short Form; QoL, quality of life; SDS, Sheehan Disability Scale.

in SDS total score and MADRS total score at week 8 was $0.5188(p<0.0001$; Table 5$)$, the change of SDS strongly correlated with MADRS change.

\section{HAM-A scores}

The mean (SD) HAM-A total score decreased significantly from 25.6 (7.01) at baseline to $8.0(7.42)$ at week 8 of treatment with escitalopram in FAS (Table 2). The remission rate based on HAM-A total score increased steadily over time. At week 8, the remission rate according to HAM-A total score $\leq 7$ was achieved in $61.3 \%$ of patients treated with escitalopram. The Spearman's rank correlation for change in SDS total score and HAM-A total score at week 8 was $0.4683(p<0.0001$; Table 5), and the correlation between change of SDS and MADRS was moderate.

\section{QIDS-SR score}

Escitalopram treatment significantly $(p<0.0001)$ decreased the mean (SD) QIDS-SR total score from 14.9 (4.29) at baseline to 6.3 (4.70) at week 8 in FAS (Table 2). The remission rate based on QIDS-SR total score increased steadily over time. By week 8 , remission was achieved in $51.5 \%$ of patients treated with escitalopram.

\section{Safety}

Overall, 25.7\% ( $n=66 / 257)$ of patients reported $\geq 1$ TEAEs during the study period. The most frequently reported TEAEs $(\geq 2 \%)$ included nausea $(5.8 \%[15 / 257])$, dizziness $(2.7 \%$ [7/257]), and diarrhea $(2.3 \%[6 / 257])$. All the TEAEs were mild to moderate in severity. Totally, four (1.6\%) patients experienced $\geq 1$ serious TEAEs and seven patients (2.7\%) discontinued treatment due to TEAEs.

In the hematology panel, abnormal laboratory values were reported for leukocytes ( $\mathrm{n}=2)$ and fasting blood glucose $(n=2)$, alanine transaminase and aspartate transaminase $(n=1$ each). Totally, three patients had an abnormal ECG at week 8 and were associated with risk factors such as old age, and had QT prolongation $<500 \mathrm{~ms}$. There were no clinically relevant changes in body weight $(-0.6 \mathrm{~kg})$, systolic blood pressure $(2 \mathrm{mmHg})$, or heart rate $(1.9 \mathrm{bpm})$ from baseline to the end of study (EOS). In the SS, 257 patients completed the C-SSRS questionnaire at baseline and 209 patients completed EOS. None of them could be classified as having positive suicidal ideation or behavior. Additionally, two $(0.8 \%, \mathrm{~N}=257)$

Table 2 Mean (SD) scores of measurement at baseline and at each visit (full analysis set, last observation carried forward)

\begin{tabular}{lllllll}
\hline Parameters & Baseline & Week I & Week 2 & Week 4 & Week 6 & Week 8 \\
\hline Q-LES-Q-SF total score & $39.3(I 4.43)$ & - & $48.8(14.29)$ & $54.3(14.76)$ & $58.2(15.47)$ & $61.6(I 5.80)$ \\
SDS total score & $17.4(5.9)$ & & $12.9(6.84)$ & $10.6(6.96)$ & $9.3(7.06)$ & $7.6(6.7 I)$ \\
$\quad$ Work & $5.9(2.31)$ & - & $4.4(2.46)$ & $3.7(2.48)$ & $3.1(2.45)$ & $2.6(2.33)$ \\
$\quad$ Social life & $6.0(2.27)$ & - & $4.3(2.42)$ & $3.5(2.37)$ & $3.1(2.39)$ & $2.5(2.32)$ \\
$\quad$ Family life & $5.6(2.30)$ & - & $4.1(2.49)$ & $3.5(2.5 I)$ & $3.0(2.54)$ & $2.5(2.42)$ \\
MADRS total score & $29.1(6.64)$ & $23.4(6.80)$ & $18.6(7.69)$ & $15.5(8.21)$ & $12.6(8.31)$ & $10.0(8.43)$ \\
HAM-A total score & $25.6(7.01)$ & $20.0(8.02)$ & $15.4(8.15)$ & $12.6(7.95)$ & $10.4(7.78)$ & $8.0(7.42)$ \\
QIDS-SR total score & $14.9(4.29)$ & $11.9(4.56)$ & $10.2(4.80)$ & $8.8(4.99)$ & $7.5(5.05)$ & $6.3(4.70)$ \\
\hline
\end{tabular}

Note: $p<0.000$ I on the change from baseline for each post-baseline visit (obtained with Wilcoxon signed-rank test).

Abbreviations: HAM-A, Hamilton Anxiety Scale; MADRS, Montgomery-Åsberg Depression Rating Scale; QIDS-SR, Quick Inventory of Depressive Symptomatology-Self Report; Q-LES-Q-SF, Quality of Life Enjoyment and Satisfaction Questionnaire-Short Form; SD, standard deviation; SDS, Sheehan Disability Scale. 
Table 3 Multiple linear regression analyses of baseline SDS total score

\begin{tabular}{lll}
\hline Variables & Coefficient & p-value \\
\hline Baseline score of Q-LES-Q-SF & 0.02758 & 0.3575 \\
Baseline score of MADRS & 0.20952 & 0.0084 \\
Baseline score of QIDS-SR & 0.40582 & 0.0005 \\
Baseline score of HAM-A & -0.00926 & 0.8737 \\
\hline
\end{tabular}

Notes: Baseline SDS score was considered as a dependent variable; multiple linear regression analyses were performed on baseline MADRS score, QIDS score, and HAM-A score.

Abbreviations: HAM-A, Hamilton Anxiety Scale; MADRS, Montgomery-Åsberg Depression Rating Scale; QIDS-SR, Quick Inventory of Depressive SymptomatologySelf Report; Q-LES-Q-SF, Quality of Life Enjoyment and Satisfaction QuestionnaireShort Form; SDS, Sheehan Disability Scale.

patients reported suicide attempts, one $(0.4, \mathrm{~N}=257)$ patient completed suicide during the study period, but no suicidal ideation or behavior was reported at the previous visit.

\section{Discussion}

The current study investigated the impact of escitalopram on social function in the treatment of Chinese MDD patients with anxiety symptoms. Escitalopram treatment (10-20 mg/day) was found to be efficacious, with no new safety signals observed in the study population.

Several randomized controlled studies have used SDS as an outcome measure to assess the impact of antidepressants on self-reported disability across a wide range of disorders, including MDD ${ }^{48}$ In the current study, SDS total score declined significantly with escitalopram treatment. As expected, the changes of SDS were strongly associated with depression severity score and moderately related to anxiety severity score. These treatment effects are consistent with those reported in an open-label study, where a mean decrease in SDS score of -10.0 was noted after 15 weeks of treatment with escitalopram $10 \mathrm{mg} /$ day. $^{49}$ The results were also compared with that of a 10-week RCT, where a mean decrease in SDS score of -11.1 was observed with levomilnacipran ER $75-100 \mathrm{mg} /$ day. ${ }^{50}$ The extent of decrease in self-rated SDS score correlated with a reduction in psychiatric symptoms (as measured with the Clinical Global Impressions Scale). ${ }^{51}$

Impact factors of baseline SDS total scores and SDS remission at the end point were investigated. Baseline SDS score was associated with depression severity but not with baseline anxiety score. These results were consistent with Sheehan et al, ${ }^{23}$ who demonstrated that residual functional impairment may persist even after resolution of depressive symptoms, indicating significant impacts of depression on symptoms of functions. In the multiple logistic regression analyses, the reduction of MADRS at week 8 , reduction of HAMA at week 2 and week 6 were the validated impact factors of SDS remission at end point. In contrast, reduction of anxiety score at an early phase of treatment strongly attributed to remission of SDS score at the end point, followed by reduction of depression score at EOS.

In parallel, Q-LES-Q-SF scores increased significantly as early as the first week of treatment, and this improvement

Table 4 Multiple logistic regression analyses of impact factors of SDS remission at end point

\begin{tabular}{|c|c|c|c|c|}
\hline Variables & OR & $\begin{array}{l}\text { Lower limit } \\
\text { of } 95 \% \mathrm{Cl}\end{array}$ & $\begin{array}{l}\text { Upper limit } \\
\text { of } 95 \% \mathrm{Cl}\end{array}$ & $p$-value \\
\hline \multicolumn{5}{|l|}{ Single-factor logistic analysis } \\
\hline Baseline Q-LES-Q-SF score & 0.992 & 0.974 & 1.011 & 0.4205 \\
\hline Baseline MADRS score & 0.968 & 0.929 & 1.008 & 0.1187 \\
\hline Baseline QIDS-SR score & 0.953 & 0.895 & 1.015 & 0.1362 \\
\hline Baseline HAM-A score & 0.983 & 0.946 & 1.021 & 0.3696 \\
\hline $\begin{array}{l}\text { Whether "live with family" (yes, } \\
n=109 / 180 \text { vs no, } n=26 / 48 \text { ) }\end{array}$ & 1.299 & 0.684 & 2.468 & 0.4242 \\
\hline Change of MADRS at week 2 & 0.964 & 0.951 & 0.978 & $<0.0001$ \\
\hline Change of MADRS at week 6 & 0.930 & 0.912 & 0.947 & $<0.0001$ \\
\hline Change of MADRS at week 8 & 0.930 & 0.912 & 0.947 & $<0.0001$ \\
\hline Change of HAM-A at week 2 & 0.962 & 0.949 & 0.975 & $<0.0001$ \\
\hline Change of HAM-A at week 6 & 0.934 & 0.917 & 0.951 & $<0.0001$ \\
\hline Change of HAM-A at week 8 & 0.932 & 0.914 & 0.951 & $<0.0001$ \\
\hline \multicolumn{5}{|l|}{ Multiple-factors logistic analysis } \\
\hline Change of MADRS at week 8 & 0.951 & 0.930 & 0.972 & $<0.000$ I \\
\hline Change of HAM-A at week 2 & 0.984 & 0.968 & 1.000 & 0.0446 \\
\hline Change of HAM-A at week 6 & 0.967 & 0.945 & 0.990 & 0.0001 \\
\hline
\end{tabular}

Notes: Remission at end point (SDS $\leq 6$ vs SDS $>6$ ) was considered as a dependent factor; baseline depression, anxiety, quality of life, "live with family" status, the reduction of MADRS and HAM-A at each visit as covariables; impact factors of SDS remission was selected by single-factor logistic analysis using a stepwise selection process and then validated by multiple-factors logistic analysis.

Abbreviations: Cl, confidence interval; HAM-A, Hamilton Anxiety Scale; MADRS, Montgomery-Åsberg Depression Rating Scale; OR, odds ratio; QIDS-SR, Quick Inventory of Depressive Symptomatology-Self Report; Q-LES-Q-SF, Quality of Life Enjoyment and Satisfaction Questionnaire-Short Form; SDS, Sheehan Disability Scale. 


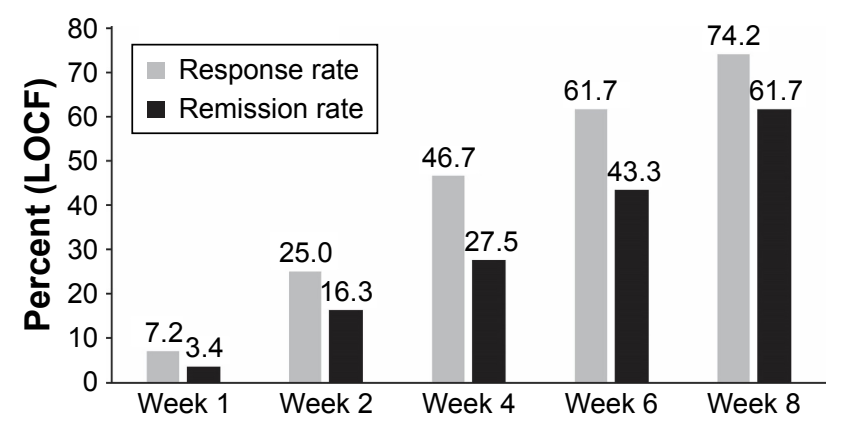

Figure 2 MADRS response and remission rate (full analysis set, last observation carried forward).

Notes: Response: $\geq 50 \%$ improvement from baseline; remission: MADRS total score $\leq 10$.

Abbreviations: LOCF, last observation carried forward; MADRS, MontgomeryÅsberg Depression Rating Scale.

continued through 8 weeks of escitalopram treatment. The mean (SD) Q-LES-Q-SF total score increased from 39.3 (14.43) at baseline to $61.6(15.80)$ at week 8 (mean increase in Q-LES-Q-SF score of 22.3 points), indicating a steady and sustained improvement in satisfaction with life. A longitudinal analysis assessing QoL in patients with MDD from four 8-week, double-blind, placebo-controlled studies reported significant improvement in Q-LES-Q-SF scores with escitalopram vs placebo. ${ }^{52}$ Additionally, $34 \%$ of escitalopramtreated patients achieved a Q-LES-Q-SF score within $10 \%$ of the community norm compared versus placebo $(27 \%))^{52}$ The improvements in Q-LES-Q-SF scores were also consistent with those reported for other antidepressants, including venlafaxine,${ }^{53}$ desvenlafaxine,${ }^{54}$ and vortioxetine. ${ }^{55}$

Table 5 Association between the change from baseline to week 8 in SDS total score and MADRS total score and HAM-A total score at each visit (full analysis set, last observation carried forward)

\begin{tabular}{|c|c|c|c|}
\hline & $\mathbf{n}$ & $\begin{array}{l}\text { Correlation } \\
\text { coefficient }\end{array}$ & $p$-value \\
\hline \multicolumn{4}{|c|}{ SDS total score and MADRS total score } \\
\hline \multicolumn{4}{|c|}{ Visit (week) } \\
\hline 2 & 216 & 0.4396 & $<0.000$ I \\
\hline 4 & 225 & 0.5358 & \\
\hline 6 & 225 & 0.5483 & \\
\hline 8 & 225 & 0.5188 & \\
\hline \multicolumn{4}{|c|}{ SDS total score and HAM-A total score } \\
\hline \multicolumn{4}{|c|}{ Visit (week) } \\
\hline 2 & 216 & 0.2989 & $<0.000$ I \\
\hline 4 & 225 & 0.4332 & \\
\hline 6 & 225 & 0.4918 & \\
\hline 8 & 225 & 0.4683 & \\
\hline
\end{tabular}

Note: Correlation coefficients were calculated according to Spearman rank correlation.

Abbreviations: HAM-A, Hamilton Anxiety Scale; MADRS, Montgomery-Åsberg Depression Rating Scale; $n$, total number of patients in a subset; SDS, Sheehan Disability Scale.
In this study, the mean MADRS scores decreased significantly as early as the first week of treatment, and this improvement persisted over 8 weeks of escitalopram treatment. The mean (SD) MADRS total score significantly decreased from baseline to week 8 of treatment with escitalopram (mean decrease in MADRS total score: -19.1 points). This is almost consistent with another study that evaluated the efficacy and tolerability of escitalopram in patients with MDD and anxiety symptoms, where the mean change from baseline in MADRS total score was -21.7 points at week $8 .{ }^{14}$ However, the current study lacks a placebo or active control group data to compare the efficacy. Analysis of the relationship between the change in SDS total score, MADRS and HAM-A total scores over 8 weeks suggests a positive correlation between improvement in SDS total score and improvement in depressive and anxiety symptoms. Similar results were also observed in a pooled analysis of patients with MDD treated with desvenlafaxine ${ }^{18}$ and in a post hoc analysis that assessed the relationship between clinical and functional outcomes in patients with MDD following a treatment switch to duloxetine. ${ }^{56}$

The mean QIDS-SR total score decreased from baseline to EOS by -8.6 points. The remission rate according to MADRS scale was higher than the QIDS-SR scale ( $61.7 \%$ vs $50.6 \%$ ). The reduction of mean HAM-A total score at week 8 from baseline was 17.6 points, indicating steady and sustained improvement in depression. However, the inter-rater reliability of the participating raters at different centers was not established for the overall data analysis.

A small sample size, open nature of the study design, and lack of placebo or active comparator group limit the possibility of comparing the efficacy of escitalopram with similar studies. Due to the open-label nature of the study, a causal relationship between escitalopram treatment and clinical improvements is difficult to establish. Further, the blood levels of escitalopram were not measured to ascertain compliance and adequate absorption of the drug.

Escitalopram treatment exhibited a good tolerability profile, with most TEAEs being mild to moderate in severity, consistent with the previous studies. ${ }^{21,31,57}$ Nausea, dizziness, and diarrhea were the most commonly reported TEAEs and are a known risk of SSRIs. ${ }^{21,58}$ Escitalopram treatment led to no overt or clinically significant changes in either body weight or BMI. Patients with comorbid depression and anxiety symptoms may experience a greater likelihood of suicidal behavior than those with anxiety alone.$^{58}$ Comorbidity with anxiety disorders was not excluded, which may also underestimate the severity of anxiety symptoms. Limited 
use of benzodiazepines during the initial treatment period may have hindered the rapid control of anxiety symptoms in the current study. Data from placebo-controlled studies in patients with MDD and anxiety disorders treated with escitalopram ${ }^{59}$ reported no indication of increased risk of suicidal behavior among escitalopram-treated patients versus placebo. Another meta-analysis of placebo-controlled studies, including 2,648 patients, reported only one instance of suicide, which occurred 6 days after cessation of escitalopram treatment. ${ }^{60}$ Nevertheless, health care professionals should monitor patients for clinical worsening and suicide risk prior to antidepressant therapy, early in the treatment period, and at the time of dose escalation.

\section{Conclusion}

Escitalopram (10-20 mg/day) treatment was efficacious in reducing depression and anxiety symptoms and improving the social function and QoL of depression in patients with comorbid anxiety. No new safety signals were observed with escitalopram over the 8-week treatment period.

\section{Acknowledgments}

The authors thank Dr Pan Jiyang for her role in enrolment of patients and conducting the study. The authors also thank the patients in this study, without whom the study would never have been accomplished. Writing assistance was provided by Dr Palash Kumar Das and additional editorial support was provided by Dr Rohit Bhandari (SIRO Clinpharm Pvt. Ltd., Thane, India), funded by Xi'an Janssen Pharmaceutical Ltd., China.

\section{Author contributions}

All authors contributed toward data analysis, drafting and revising the paper and agree to be accountable for all aspects of the work. The sponsor also provided a formal review of this manuscript. All authors met ICMJE criteria and all those who fulfilled those criteria are listed as authors. All authors had access to the study data and made the final decision about where to publish these data and approved submission to this journal.

\section{Disclosure}

The study presented in this report was sponsored by Xi'an Janssen Pharmaceutical Ltd. JW is an employee of Lundbeck Pharmaceutical Information Consulting Co., Ltd., China, JZ, and CH are employees of Xi' an Janssen Pharmaceutical Ltd., China. The authors report no other conflicts of interest in this work.

\section{References}

1. Ferrari AJ, Charlson FJ, Norman RE, et al. Burden of depressive disorders by country, sex, age, and year: findings from the global burden of disease study 2010. PLoS Med. 2013;10(11):e1001547.

2. Global Burden of Disease Study 2013 Collaborators. Global, regional, and national incidence, prevalence, and years lived with disability for 301 acute and chronic diseases and injuries in 188 countries, 1990-2013: a systematic analysis for the global burden of disease study 2013. Lancet. 2015;386(9995):743-800.

3. Lam RW. Depression, Revised. 2nd ed. Oxford University Press; 2012. Available from http://oxfordmedicine.com/view/10.1093/ med/9780199692736.001.0001/med-9780199692736. Accessed June 7, 2018.

4. Gupta S, Goren A, Dong P, Liu D. Prevalence, awareness, and burden of major depressive disorder in urban China. Expert Rev Pharmacoecon Outcomes Res. 2016;16(3):393-407.

5. Gu L, Xie J, Long J, et al. Epidemiology of major depressive disorder in mainland china: a systematic review. PLoS One. 2013;8(6):e65356.

6. Birnbaum HG, Kessler RC, Kelley D, Ben-Hamadi R, Joish VN, Greenberg PE. Employer burden of mild, moderate, and severe major depressive disorder: mental health services utilization and costs, and work performance. Depress Anxiety. 2010;27(1):78-89.

7. Hu TW, He Y, Zhang M, Chen N. Economic costs of depression in China. Soc Psychiatry Psychiatr Epidemiol. 2007;42(2):110-116.

8. Evans-Lacko S, Knapp M. Global patterns of workplace productivity for people with depression: absenteeism and presenteeism costs across eight diverse countries. Soc Psychiatry Psychiatr Epidemiol. 2016;51(11): $1525-1537$.

9. Wu Z, Fang Y. Comorbidity of depressive and anxiety disorders: challenges in diagnosis and assessment. Shanghai Arch Psychiatry. 2014; 26(4):227-231.

10. Adams GC, Balbuena L, Meng X, Asmundson GJ. When social anxiety and depression go together: a population study of comorbidity and associated consequences. $J$ Affect Disord. 2016;206:48-54.

11. Xin LM, Chen L, Ji ZP, et al. Risk factors for anxiety in major depressive disorder patients. Clin Psychopharmacol Neurosci. 2015;13(3):263-268.

12. Shi SS, Zhang MY, Wu WY, et al. Multi-center study of the clinical features in depression comorbidity with anxiety disorders. Shanghai Arch Psychiatry. 2009;21(4):198-202.

13. deHeer EW, Gerrits MM, Beekman AT, et al. The association of depression and anxiety with pain: a study from NESDA. PLoS One. 2014; 9(10):e106907.

14. Jiang K, Li L, Wang X, et al. Efficacy and tolerability of escitalopram in treatment of major depressive disorder with anxiety symptoms: a 24-week, open-label, prospective study in Chinese population. Neuropsychiatr Dis Treat. 2017;13:515-526.

15. Saveanu R, Etkin A, Duchemin AM, et al. The international Study to predict optimized treatment in depression (iSPOT-D): outcomes from the acute phase of antidepressant treatment. J Psychiatr Res. 2015;61:1-12.

16. Fava M, Rush AJ, Alpert JE, et al. Difference in treatment outcome in outpatients with anxious versus nonanxious depression: a STAR*D report. Am J Psychiatry. 2008;165(3):342-351.

17. Pfeiffer PN, Ganoczy D, Ilgen M, Zivin K, Valenstein M. Comorbid anxiety as a suicide risk factor among depressed veterans. Depress Anxiety. 2009;26(8):752-757.

18. Guico-Pabia CJ, Fayyad RS, Soares CN. Assessing the relationship between functional impairment/recovery and depression severity: a pooled analysis. Int Clin Psychopharmacol. 2012;27(1):1-7.

19. Gili M, García Toro M, Armengol S, García-Campayo J, Castro A, Roca M. Functional impairment in patients with major depressive disorder and comorbid anxiety disorder. Can J Psychiatry. 2013;58(12):679-686.

20. Papakostas GI. Major depressive disorder: psychosocial impairment and key considerations in functional improvement. Am J Manag Care. 2009;15(11 Suppl):S316-S321.

21. Gureje O, Ademola A, Olley BO. Depression and disability: comparisons with common physical conditions in the Ibadan study of aging. $J$ Am Geriatr Soc. 2008;56(11):2033-2038. 
22. Nakano $\mathrm{Y}$, Baba $\mathrm{H}$, Maeshima $\mathrm{H}$, et al. Executive dysfunction in medicated, remitted state of major depression. J Affect Disord. 2008;111(1): $46-51$.

23. Sheehan DV, Harnett-Sheehan K, Spann ME, Thompson HF, Prakash A. Assessing remission in major depressive disorder and generalized anxiety disorder clinical trials with the discan metric of the Sheehan disability scale. Int Clin Psychopharmacol. 2011;26(2):75-83.

24. Zimmerman M, McGlinchey JB, Posternak MA, Friedman M, Attiullah N, Boerescu D. How should remission from depression be defined? The depressed patient's perspective. Am J Psychiatry. 2006;163(1):148-150.

25. Pintor L, Torres X, Navarro V, Matrai S, Gastó C. Is the type of remission after a major depressive episode an important risk factor to relapses in a 4-year follow up? J Affect Disord. 2004;82(2):291-296.

26. Romera I, Pérez V, Ciudad A, et al. Residual symptoms and functioning in depression, does the type of residual symptom matter? A post-hoc analysis. BMC Psychiatry. 2013;13:51.

27. Sheehan DV, Nakagome K, Asami Y, Pappadopulos EA, Boucher M. Restoring function in major depressive disorder: a systematic review. J Affect Disord. 2017;215:299-313.

28. Rush AJ, Kraemer HC, Sackeim HA, et al; ACNP Task Force. Report by the ACNP task force on response and remission in major depressive disorder. Neuropsychopharmacology. 2006;31(9):1841-1853.

29. Sobocki P, Ekman M, Agren H, Runeson B, Jönsson B. The mission is remission: health economic consequences of achieving full remission with antidepressant treatment for depression. Int J Clin Pract. 2006; 60(7):791-798.

30. Coplan JD, Aaronson CJ, Panthangi V, Kim Y. Treating comorbid anxiety and depression: psychosocial and pharmacological approaches. World J Psychiatry. 2015;5(4):366-378.

31. Lepola UM, Loft H, Reines EH. Escitalopram (10-20 mg/day) is effective and well tolerated in a placebo-controlled study in depression in primary care. Int Clin Psychopharmacol. 2003;18(4):211-217.

32. Bielski RJ, Ventura D, Chang CC. A double-blind comparison of escitalopram with venlafaxine extended release in the treatment of major depressive disorder. J Clin Psychiatry. 2004;65(9):1190-1196.

33. Kennedy SH, Andersen HF, Lam RW. Efficacy of escitalopram in the treatment of major depressive disorder compared with conventional selective serotonin reuptake inhibitors and venlafaxine XR: a metaanalysis. J Psychiatry Neurosci. 2006;31(2):122-131.

34. Khoo AL, Zhou HJ, Teng M, et al. Network meta-analysis and costeffectiveness analysis of new generation antidepressants. CNS Drugs. 2015;29(8):695-712.

35. Olié JP, Tonnoir B, Ménard F, Galinowski A. A prospective study of escitalopram in the treatment of major depressive episodes in the presence or absence of anxiety. Depress Anxiety. 2007;24(5):318-324.

36. Sheehan DV, Mancini M, Wang J, et al. Assessment of functional outcomes by Sheehan Disability Scale in patients with major depressive disorder treated with duloxetine versus selective serotonin reuptake inhibitors. Hum Psychopharmacol. 2016;31(1):53-63.

37. Wade AG, Fernández JL, François C, Hansen K, Danchenko N, Despiegel N. Escitalopram and duloxetine in major depressive disorder: a pharmacoeconomic comparison using UK cost data. Pharmacoeconomics. 2008;26(11):969-981.

38. Association AP. Diagnostic and Statistical Manual of Mental Disorders (DSM). Washington, DC: American Psychiatric Association; 1994:143-147.

39. Sheehan Disability Scale (SDS) - Overview. Available from: www. cqaimh.org/pdf/tool_lof_sds.pdf. Accessed January 2, 2018.

40. Endicott J, Nee J, Harrison W, et al. Quality of life enjoyment and satisfaction questionnaire: a new measure. Psychopharmacol Bull. 1993;29(2):321-326.

41. Gérard A, Liard F, Crochard A, Goni S, Millet B. Disability in patients consulting for anxiety or mood disorders in primary care: response to antidepressant treatment. Neuropsychiatr Dis Treat. 2012;8:605-614.
42. IsHak WW, Mirocha J, Pi S, et al. Patient-reported outcomes before and after treatment of major depressive disorder. Dialogues Clin Neurosci. 2014;16(2):171-183.

43. Montgomery SA, Asberg M. A new depression scale designed to be sensitive to change. Br J Psychiatry. 1979;134:382-389.

44. Sajatovic M, Ramirez LF. Hamilton anxiety scale (HAM-A). In: Rating Scales in Mental Health. 3rd ed. Baltimore, USA: The John Hopkins University Press; 2012.

45. Rush AJ, Trivedi MH, Ibrahim HM, et al. The 16-item quick inventory of depressive symptomatology (QIDS), clinician rating (QIDS-C), and self-report (QIDS-SR): a psychometric evaluation in patients with chronic major depression. Biol Psychiatry. 2003;54(5):573-583.

46. Cohen J. Statistical Power Analysis for the Behavioral Sciences, 2nd ed. New York, NY: Academia Press; 1988.

47. Dunlop BW, Li T, Kornstein SG, et al. Correlation between patient and clinician assessments of depression severity in the PREVENT study. Psychiatry Res. 2010;177(1-2):177-183.

48. Sheehan KH, Sheehan DV. Assessing treatment effects in clinical trials with the discan metric of the Sheehan Disability Scale. Int Clin Psychopharmacol. 2008;23(2):70-83.

49. Kaul V, Dutta S, Beg MA, et al. Comparative evaluation of amisulpride and escitalopram on Sheehan's disability scale among depression patients in a tertiary care teaching hospital in Nepal. J Drug Deliv Ther. 2015;5:3-8.

50. Montgomery SA, Mansuy L, Ruth A, Bose A, Li H, Li D. Efficacy and safety of levomilnacipran sustained release in moderate to severe major depressive disorder: a randomized, double-blind, placebo-controlled, proof-of-concept study. J Clin Psychiatry. 2013;74(4):363-369.

51. Bandelow B, Baldwin DS, Dolberg OT, Andersen HF, Stein DJ. What is the threshold for symptomatic response and remission for major depressive disorder, panic disorder, social anxiety disorder, and generalized anxiety disorder? J Clin Psychiatry. 2006;67(9): $1428-1434$.

52. Demyttenaere K, Andersen HF, Reines EH. Impact of escitalopram treatment on quality of life enjoyment and satisfaction questionnaire scores in major depressive disorder and generalized anxiety disorder. Int Clin Psychopharmacol. 2008;23(5):276-286.

53. Trivedi MH, Dunner DL, Kornstein SG, et al. Psychosocial outcomes in patients with recurrent major depressive disorder during 2 years of maintenance treatment with venlafaxine extended release. $J$ Affect Disord. 2010;126(3):420-429.

54. Endicott J, Lam RW, Hsu MA, Fayyad R, Boucher M, Guico-Pabia CJ. Improvements in quality of life with desvenlafaxine $50 \mathrm{mg} / \mathrm{d}$ vs placebo in employed adults with major depressive disorder. $J$ Affect Disord. 2014;166:307-314.

55. Florea I, Danchenko N, Brignone M, Loft H, Rive B, Abetz-Webb L. The effect of vortioxetine on health-related quality of life in patients with major depressive disorder. Clin Ther. 2015;37(10): 2309-2323.

56. Sheehan DV, Chokka PR, Granger RE, Walton RJ, Raskin J, Sagman D. Clinical and functional outcomes in patients with major depressive disorder and painful physical symptoms switched to treatment with duloxetine. Hum Psychopharmacol. 2011;26(3):242-251.

57. Si T, Wang G, Yang F, et al. Efficacy and safety of escitalopram in treatment of severe depression in Chinese population. Metab Brain Dis. 2017;32(3):891-901.

58. Hranov LG. Comorbid anxiety and depression: illumination of a controversy. Int J Psychiatry Clin Pract. 2007;11(3):171-189.

59. Pedersen AG. Escitalopram and suicidality in adult depression and anxiety. Int Clin Psychopharmacol. 2005;20(3):139-143.

60. Gunnell D, Saperia J, Ashby D. Selective serotonin reuptake inhibitors (SSRIs) and suicide in adults: meta-analysis of drug company data from placebo controlled, randomised controlled trials submitted to the MHRA's safety review. BMJ. 2005;330(7488):385. 
Neuropsychiatric Disease and Treatment

Dovepress

\section{Publish your work in this journal}

Neuropsychiatric Disease and Treatment is an international, peerreviewed journal of clinical therapeutics and pharmacology focusing on concise rapid reporting of clinical or pre-clinical studies on a range of neuropsychiatric and neurological disorders. This journa is indexed on PubMed Central, the 'PsycINFO' database and CAS,

and is the official journal of The International Neuropsychiatric Association (INA). The manuscript management system is completely online and includes a very quick and fair peer-review system, which is all easy to use. Visit http://www.dovepress.com/testimonials.php to read real quotes from published authors.

Submit your manuscript here: http://www.dovepress.com/neuropsychiatric-disease-and-treatment-journal 